CERN-PPE/94-168

October 11, 1994

\title{
Search for Heavy Isosinglet Neutrinos
}

\author{
The CHARM II Collaboration
}

\author{
P. Vilain ${ }^{1}$, G. Wilquet ${ }^{1)}$ \\ Inter-University Institute for High Energies (ULB-VUB), Brussels, Belgium
}
S. Petrak ${ }^{2)}$, R. Beyer ${ }^{3)}$, W. Flegel, H. Grote, T. Mouthuy ${ }^{4)}$, H. Øveras, J. Panman, K. Winter, G. Zacek ${ }^{5)}$, V. Zacek ${ }^{6}$ CERN, Geneva, Switzerland

F. W. Büsser, C. Foos, L. Gerland, T. Layda ${ }^{7}$, F. Niebergall, G. Rädel ${ }^{3)}$, P. Stähelin, T. Voss ${ }^{3)}$ II. Institut für Experimentalphysik ${ }^{a}$, Universität, Hamburg, Germany

\author{
D. Favart, G. Grégoire, E. Knoops ${ }^{8)}$, V. Lemaître \\ Université Catholique de Louvain, Louvain-la-Neuve, Belgium
}

P. Gorbunov, E. Grigoriev, V. Khovansky, A. Maslennikov, A. Rozanov

Institute for Theoretical and Experimental Physics, Moscow, Russian Federation

\author{
W. Lippich, A. Nathaniel ${ }^{9}$, A. Staude, J. Vogt \\ Sektion Physik ${ }^{\text {a) }}$ der Universität München, Germany
}

\author{
A. G. Cocco, A. Ereditato, G. Fiorillo, F. Marchetti-Stasi, V. Palladino, P. Strolin \\ Università and Istituto Nazionale di Fisica Nucleare (INFN), Naples, Italy
}

A. Capone, D. De Pedis, U. Dore, A. Frenkel-Rambaldi, P. F. Loverre, D. Macina ${ }^{\text {9) }}$, G. Piredda, R. Santacesaria

Università 'La Sapienza' and Istituto Nazionale di Fisica Nucleare (INFN), Rome, Italy

E. Di Capua, S. Ricciardi, B. Saitta

Università di Ferrara and Istituto Nationale di Fisica Nucleare (INFN), Ferrara, Italy

\section{B. Akkus ${ }^{10)}$, E. Arik ${ }^{10)}$, M. Serin-Zeyrek, R. Sever, P. Tolun}

High Energy Physics Research Centre, YEFAM, Ankara, Turkey

K. Hiller, R. Nahnhauer, H. E. Roloff

DESY - Institut für Hochenergiephysik, Zeuthen, Germany

(submitted to Physics Letters B)

\begin{abstract}
A new search for decays of heavy isosinglet neutrinos produced by neutral-current neutrino interactions in the CERN wide-band neutrino beam has been conducted by the CHARM-II Collaboration. We searched for heavy neutrinos created by scattering muon neutrinos on nucleons and decaying into $\mu^{+} \mu^{-} \nu_{\mu}$. Production and decay of heavy neutrinos would appear as double events in the detector. Each double event candidate was tested wheather the measured quantities are compatible with the kinematics of this process. No event passed this test. The analysis is based on $2 \times 10^{7}$ neutral-current neutrino events collected from 1987-1991. The experiment is sensitive to heavy neutrinos in the mass range $0.3-2.4 \mathrm{GeV} / \mathrm{c}^{2}$. The best limit for the mixing parameter is $\left|U_{\mu i}\right|^{2}<3 \times 10^{-5}$ for a mass around $2 \mathrm{GeV} / \mathrm{c}^{2}$. The analysis improved by an order of magnitude previous results derived from wide-band neutrino beam exposures of the CHARM detector.
\end{abstract}


This heavy neutrino search is one in a variety of experiments exploring which neutrino states exist in nature. Different experimental techniques are sensitive to different types of heavy neutrinos, mass ranges and coupling strengths. The LEP experiments [1] have derived the number of light neutrino families $N_{\nu}=$ $2.90 \pm 0.027$ from a measurement of the invisible $\mathrm{Z}$ decay width. However, neutrinos which occur as singlets under the standard $S U(2)_{L}$ group, for instance right handed neutrinos, don't contribute to the $\mathrm{Z}$ decay width. Isosinglet neutrinos have no weak interactions except those induced by mixing with isodoublet neutrinos. Experimental searches for heavy isosinglet neutrinos $\nu_{h}$ which couple to muon neutrinos $\nu_{\mu}$ have been performed in meson decay experiments: $\pi \rightarrow \mu \nu_{h}$ [2], $K \rightarrow \mu \nu_{h}$ [3], $D \rightarrow \mu \nu_{h}$ [4, 5, 6] and in $e^{+} e^{-}$experiments: $e^{+} e^{-} \rightarrow Z \rightarrow \nu_{\mu} \nu_{h}[7]$

The sensitivity of the meson decay experiments is limited by the mass of the parent mesons, whereas one of the $e^{+} e^{-}$experiments [7] extended stringent limits for neutrino masses above $3 \mathrm{GeV} / \mathrm{c}^{2}$. The intermediate mass region from 0.3 to $2.4 \mathrm{GeV} / \mathrm{c}^{2}$ is covered by muon neutrino scattering experiments on nucleons: $\nu_{\mu} N \rightarrow \nu_{h} X$, where an accelerator neutrino beam is used as muon neutrino source. Former experiments $[5,8]$ already searched for heavy neutrinos produced in this way. The result presented here is based on a new method to separate signal from background events applied to a statistical sample which is 100 times larger.

The creation of heavy neutrinos $\nu_{h}$ by $\nu N$-scattering depends from the existence of neutral current transitions $\nu_{\mu} \rightarrow \nu_{h}$. Mixing between isosinglet and isodoublet neutrinos allows these transitions of different neutrino mass eigenstates in neutral current reactions. The coupling of $\nu_{h}$ to the muon neutrino $\nu_{\mu}$ is described by a mixing parameter $U$ relative to the Fermi-coupling strength.

\section{Concept of the experiment}

The CHARM-II detector and its characteristics have been described in detail in ref. [9, 10]. Double vertex events where a neutral current (NC) event is followed by a decay topology are the signature for unstable neutrinos produced in a neutrino interaction. The sensitivity of the experiment can be optimized by the choice of the decay channel. The most favourable decay mode is $\nu_{h} \rightarrow \mu^{+} \mu^{-} \nu_{\mu}$ for which the ratio of signal to background is the largest. The hadronic decay mode $\nu_{h} \rightarrow$ hadrons $+\nu_{\mu}$ has a larger branching ratio but also very large background. Such background may arise from random overlays of two independent neutrino interactions in the sensitive time of the detector and from double events due to the interaction of neutral hadrons or the decay of neutral kaons originating from a neutral current event. According to the measured event rates of interactions producing a hadron shower or a muon pair we expect for the $\mu^{+} \mu^{-} \nu_{\mu}$ decay channel a $10^{5}$ times lower background of random overlays as compared to the hadronic decay mode. The background contribution from neutral hadrons is negligible for the two muon topology, however it is an important background for neutrino decays into hadrons.

We searched for the $\mu^{+} \mu^{-} \nu_{\mu}$ decay signature, where the dimuon decay vertex is separated from the NC shower of the production process. The predicted branching ratio in the mass range of interest here is of the order of 5-7\% [11]. In a first step we applied geometrical cuts to collect a candidate data sample. These candidate events were then subjected to a kinematical fit to dismiss uncorrelated double events. The kinematics of $\nu_{h}$ production and decay is constrained by eight equations of energy and momentum conservation at both vertices. The equations include unmeasured quantities, corresponding to the three invisible neutrinos involved in the process: the incoming muon neutrino, the heavy neutrino and a muon neutrino in the final state. The direction of the heavy neutrino is given by the line connecting the vertices of both subevents. There are therefore six unknown variables, one for the energy of the incoming neutrino, the mass and the energy of the heavy neutrino and three for the momentum vector of the neutrino among the decay products. The system is thus overdetermined by two degrees of freedom and a fit based on the minimum $\chi^{2}$ method is performed to test the kinematical hypothesis.

\footnotetext{
1) National Foundation for Scientific Research, Belgium

2) Freie Universität, Berlin, Germany

3) Now at DESY, Hamburg, Germany

4) Now at Centre de Physique des Particules de Marseille, Faculté de Luminy, Marseille, France

5) Now at McGill University, Montreal, Canada

6) Now at Université de Montreal, Montreal, Canada

7) Now at University of California at Santa Cruz, USA

8) Inter-University Institute for Nuclear Science, Belgium

9) Now at CERN, Geneva, Switzerland

10) Bogaziçi University, Istanbul, Turkey

a) supported by the German Bundersministerium für Forschung und Technologie (BMFT), under contract numbers 05-5HH22P and 05-5MU12P
} 


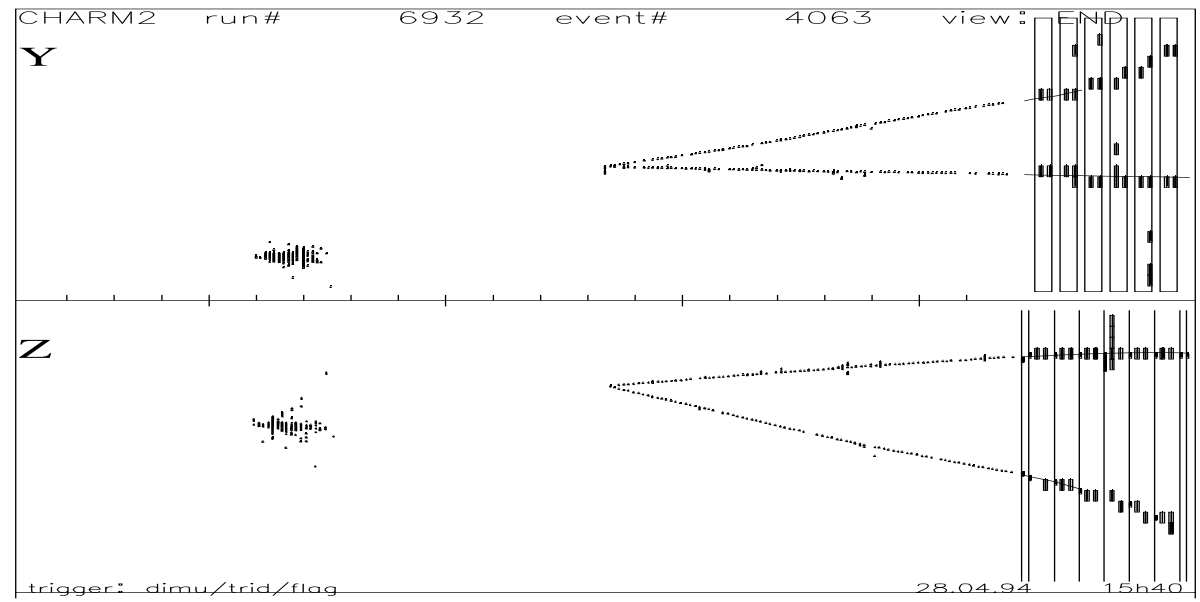

Figure 1: One candidate event selected with the geometrical cuts and before the kinematical fit was applied.

\section{Event selection}

The original data sample consisted of events selected by a two muon trigger. The geometrical selection criteria are applied to the hits of the streamer tubes. The following conditions were used to select the candidate event sample: (i) The interaction and decay point were required to be in a fiducial region of a square, $320 \times$ $320 \mathrm{~cm}^{2}$, transversely to the beam direction and longitudinally between plane 5 and 390 out of 420 calorimeter planes. (ii) The visible total energy of the hadronic shower was required to be larger than $2 \mathrm{GeV}$. The shower was classified as a $\mathrm{NC}$ event if all track candidates are shorter than $3.2 \mathrm{~m}$, equivalent to an energy loss of 1.4 $\mathrm{GeV}$. (iii) In order to ensure the separation of the two subevents we required 4 empty planes between the end of the shower and the dimuon vertex in two projections. (iv) A single muon vertex is defined as the first hit which can unambiguously be attached to the fitted muon track. Two muons were considered to have a common vertex if the three-dimensional spatial difference between individual muon vertices is smaller than $50 \mathrm{~cm}$. (v) Muons were identified by requiring a minimal track length of 80 planes, corresponding to $1.7 \mathrm{GeV}$ energy loss. Muons produce additional hits by bremsstrahlung and by $\delta$-rays, whereas hadrons interact and produce backscattered tracks and wide showers. On the basis of these differences we have attempted to eliminate dimuon events induced by neutrinos with hadronic activity at the vertex. The quantity used to pick out muon tracks is the number of additional hits. A hit is considered as additional if the distance to the predicted track position is greater than $10 \mathrm{~cm}$ and smaller than $50 \mathrm{~cm}$. We studied the additional hit distribution on real events with a $\mu \mu$ and $\mu \pi$ final state. We could suppress the hadron background while keeping the muon efficiency high if we restrict the activity in the first 20 planes to 7 additional hits and the ratio of total additional activity to track length to 0.15 additional hits per plane.

One candidate event selected by the geometrical conditions is shown in fig. 1. In a second step, the compatibility of the event kinematics with that of $\nu_{h}$ production and decay has been tested using the $\chi^{2}$ fit described above.

The validity of the kinematical fit was tested with Monte Carlo (MC) events. Events, which satisfy the kinematical hypothesis and for which the uncertainties of the measurements are correctly determined are characterized by values of $\chi^{2}$ found by the minimization which are distributed like a $\chi^{2}$ function. The $\chi^{2}$ distribution was confirmed for samples of simulated heavy neutrino events of different mass and mixing parameters. Events were selected by a cut in probability at a level of $1 \%$.

A new method was developed to determine the invariant mass of a hadronic shower. The method is based on the correlation between the invariant shower mass W and the energy transverse to the shower direction $E_{\text {trans }}$. The energy $E_{\text {trans }}$ is given by the product of energy and opening angle of the shower. The shower profiles in each plane were added up along the shower direction until $90 \%$ of all hits were used. The ratio of the mean width of this distribution and the distance from shower vertex to the center of the shower energy defines the opening angle. The functional dependence of the invariant shower mass $\mathrm{W}$ on the transverse energy $E_{\text {trans }}$ was determined in charged current events where the kinematics is constrained by the muon momentum measured in the spectrometer. The fractional resolution for the invariant shower mass is shown in fig. 2.

In table 1 the reduction of candidates at different stages of the analysis is summarized for real and for $\mathrm{MC}$ events. The number of selected MC events was normalized to the the total number of $1.2 \times 10^{7}$ neutrino and $0.9 \times 10^{7}$ antineutrino $\mathrm{NC}$ events which were combined in our analysis, the MC statistics was 1000 events at the level of the kinematical fit. 


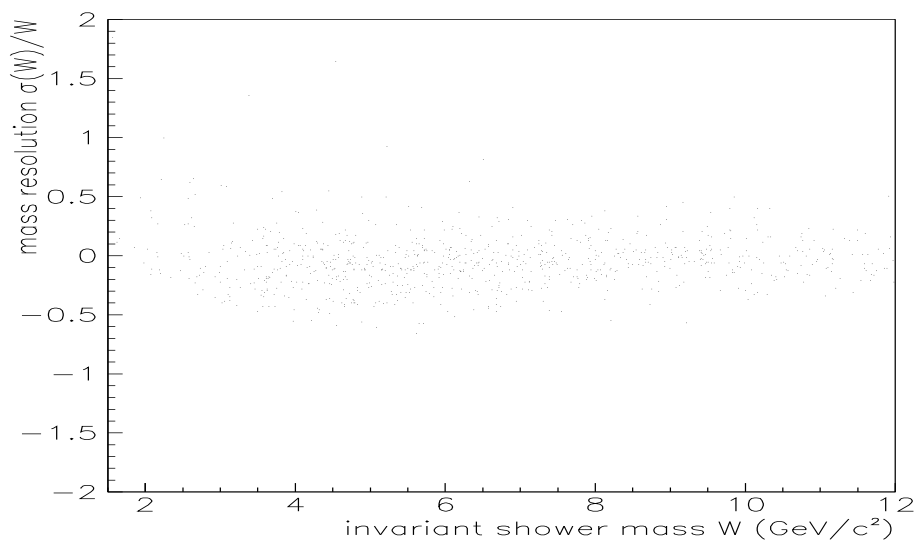

Figure 2: Fractional resolution of the measured invariant shower mass $\sigma(W) / W$ as a function of $\mathrm{W}$.

In a first selection a sample of 38504 events with a $\mu \mu$ or $\mu \pi$ state and little hadronic activity was collected. From these, 121 events were recognized as double events with a NC shower in front of the track pair. Applying the geometrical cuts the event number was reduced to ten. These ten events, one of which is shown in figure 1 constitute the candidate sample. In four events the time difference between the primary shower and the two muons was less than the gate time of the scintillation counters of $\approx 150 \mathrm{nsec}$.

We estimated the background due to random overlays by comparing the event numbers in the gate time of the scintillators and of the streamer tubes. Scaling the total event number ten according to the ratio of gate times for the scintillators and the streamer tubes we estimated a mean number $2.6 \pm{ }_{0.2}^{0.7}$ of uncorrelated double events in the scintillator time gate. The four observed events in the scintillator gate are thus consistent with random overlays within one standard deviation.

The number of ten events in the candidate sample is also fully consistent with two independent neutrino interactions yielding a NC shower or a two muon topology. We determined the number of single events that passed all geometrical criteria for a muon pair. The number of events with $E_{S h}>2 G e V$ was used to calculate the probability that a NC event occurs in the signal time of the streamer tubes. From this estimation we expect $10 \pm 4$ background events due to random superposition.

It is the advantage of the kinematical fit method that it can reduce the background to zero event. Applying it to the events in the candidate sample no event was found with a $\chi^{2}$ probability above $1 \%$.

Table 1: Real event numbers at different stages of the analysis in comparison with the numbers of a simulated neutrino of mass $\mathrm{m}=2 \mathrm{GeV} / \mathrm{c}^{2}$ and mixing $|U|^{2}=10^{-4}$ corresponding to $2.1 \times 10^{7} \nu_{\mu}$ and $\bar{\nu}_{\mu} \mathrm{NC}$ events.

\begin{tabular}{|l|r|r|}
\hline topology & real events & MC events \\
\hline two tracks & 38504 & 5.1 \\
\hline NC and two tracks & 121 & 4.6 \\
\hline NC and two muons & 10 & 3.75 \\
\hline kin. fit o.k. & 0 & 3.75 \\
\hline
\end{tabular}

\section{Sensitivity}

The expected number $\mathrm{N}$ of decays of heavy neutrinos with mass $\mathrm{m}$ and mixing parameter $\mathrm{U}$ was computed according to the expression:

$$
N=N_{\nu_{h}}\left(m,|U|^{2}\right) B\left(\nu_{h}(m) \rightarrow \mu \mu \nu\right) \epsilon\left(m,|U|^{2}\right)
$$

where $N_{\nu_{\boldsymbol{h}}}\left(m,|U|^{2}\right)$ is the number of heavy neutrinos produced in a NC neutrino interaction. It was obtained from the total number of $\mathrm{NC}$ events observed during the exposure times the square of the mixing parameter $|U|^{2}$ and taking into account the threshold effect due to the heavy neutrino mass.

$$
N_{\nu_{h}}\left(m,|U|^{2}\right)=N_{N C} \cdot|U|^{2} \cdot \sigma\left(m_{\nu_{h}}\right) / \sigma(m=0) .
$$




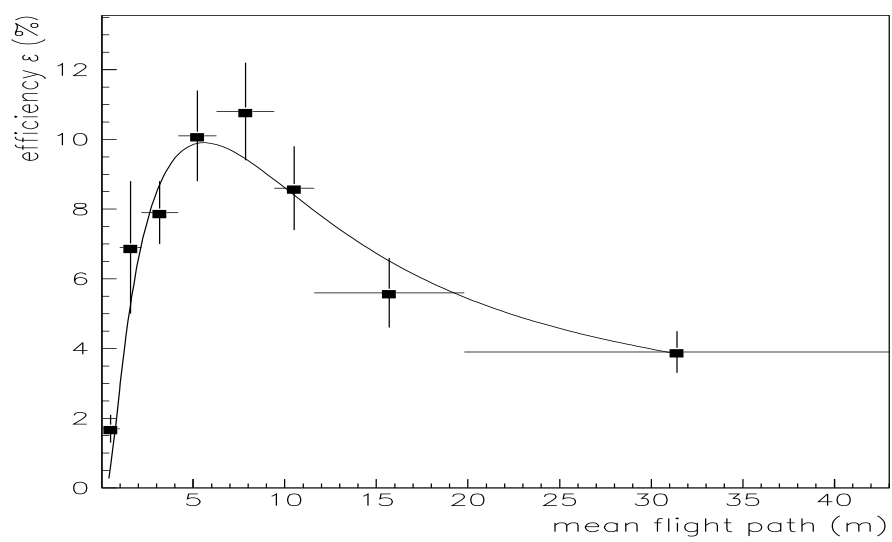

Figure 3: Efficiency $\epsilon$ to select heavy neutrino events as a function of the mean flight path.

The probability to observe the neutrino decay is the product of the branching ratio $B\left(\nu_{h}(m) \rightarrow \mu \mu \nu\right)$ and the efficiency $\epsilon\left(m,|U|^{2}\right)$ to recognize events by their topology and kinematics in the fiducial volume of the detector. The suppression factor $|U|^{2}$ for the decay process enters in the mean lifetime of the heavy neutrino.

A full MC simulation of the process was used to estimate the efficiency as a function of the heavy neutrino parameters. The efficiency was computed for different combinations of hadronic shower energies and relative distances between the two subevents. The efficiency values were fitted as a function of the mean flight path with a theoretical approach given by the probability that heavy neutrino production and decay occur within the detector length $\mathrm{L}$ and separated by a gap of length 1 , where a uniform distribution of production vertices over the detector length $\mathrm{L}$ and an exponential flight path distribution is assumed [11]. Double vertex events can be detected with a minimal vertex distance $1=1.5 \mathrm{~m}$ ( 4 empty planes in both projections) over an effective detector length of L=30 m. Fig. 3 shows the efficiency as a function of the mean flight path. The maximum efficiency of $10 \%$ occurs at about $6 \mathrm{~m}$ mean flight path.

Having observed no heavy neutrino candidate event, the $90 \%$ confidence limit on $\left|U_{\mu h}\right|^{2}$ was computed by inserting $\mathrm{N}=2.3$ into eq. (2). Systematic uncertainties affecting the efficiency $\epsilon\left(m,|U|^{2}\right)$ in eq. (1), because of systematic errors in the definition of the shower energy scale, the muon energy scale and the minimum separation of the two subevents, and the number of heavy neutrinos $N_{\nu_{h}}$ in eq (1) because of monitoring uncertainties, have been evaluated and amount to $7.8 \%$. The result is shown in fig. 4 , together with the results of earlier searches.

In conclusion, there is no evidence for heavy neutrinos in the CHARM-II data. A heavy neutrino with mass in the range 0.3 to $2.4 \mathrm{GeV} / \mathrm{c}^{2}$ for a coupling to muon neutrinos between $1 \times 10^{-2}$ and $3 \times 10^{-5}$ of the Fermi strength is excluded at the $90 \%$ confidence level.

The analysis improves a previous result from a double event analysis in the CHARM detector [5] by an order of magnitude. The mass region from 0.4 to $1.9 \mathrm{GeV} / \mathrm{c}^{2}$ is also covered by the beam-dump experiments $[4,5,6]$ studying the decay $D \rightarrow \mu \nu_{h}$. However, both kinds of experiments differ in the typical decay path of the heavy neutrinos and are therefore sensitive to different mixing parameters. The sensitive region is determined by a lower limit of $|U|^{2}$ given by the rate of production of heavy neutrinos and an upper limit of $|U|^{2}$ owing to the rapid decay of the heavy neutrinos.

\section{$5 \quad$ Acknowledgements}

We gratefully acknowledge the help of our many technical collaborators who have contributed to the realisation and the operation of the detector We are grateful for the grants from the Inter-University Institute for Nuclear Sciences (Belgium), CERN (Geneva, Switzerland), the Bundesministerium fur Forschung und Technologie FRG), the Institute of Theoretical and Experimental Physics (Moscow, Russian Federation), the Istituto Nazionale di Fisica Nucleare (Italy), and the Turkish Scientific and Technical Research Council (TUBITAK); which made the experiment possible. We should like to thank the CERN SPS operating crew and the neutrino beam staff for their competent assistance ensuring the excellent performance of their facilities. 


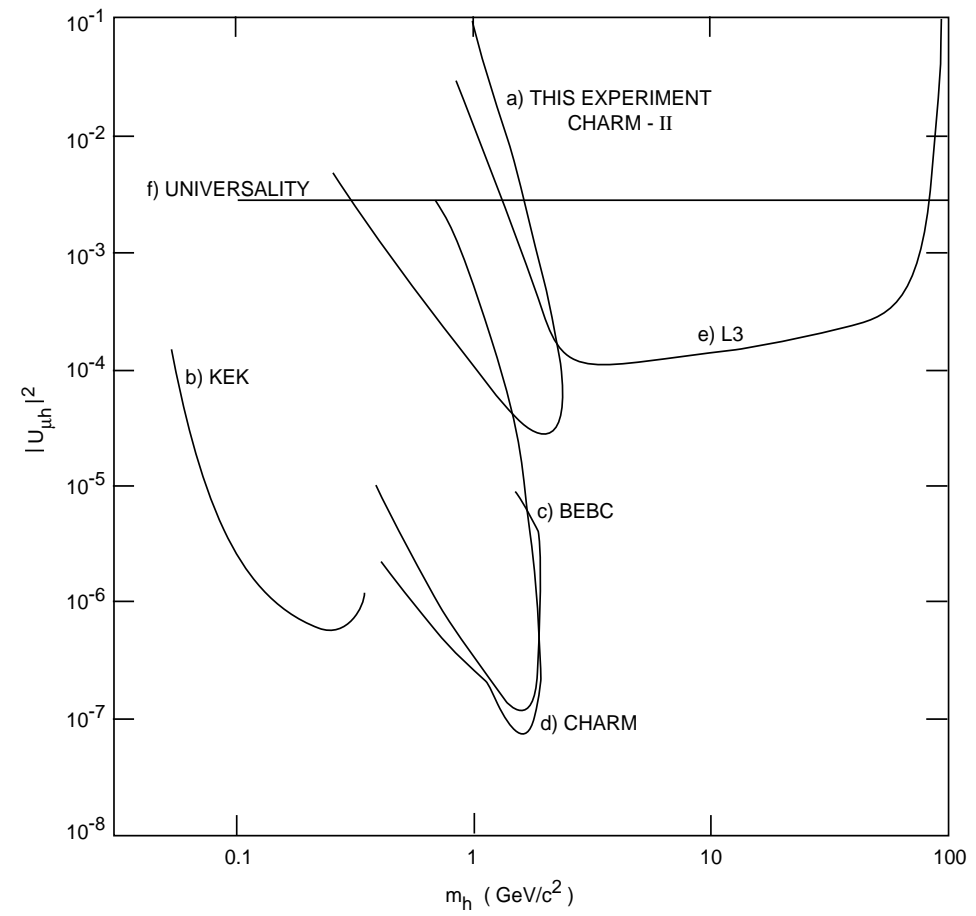

Figure 4: Limits at 90\% confidence level on $\left|U_{\mu h}\right|^{2}$ as a function of the isosinglet neutrino mass $m_{h}$ : a) limits obtained in this experiment by the CHARM II Collaboration; b) limits from the study of the decay $K \rightarrow \mu \nu$ at KEK [3]; c) limits obtained by the BEBC Collaboration in a beam-dump experiment [4]; d) limits obtained in a previous beam-dump experiment by the CHARM Collaboration [5]; e) $95 \%$ confidence limit from Z decay obtained by the L3 Collaboration [7]; f) universality limit [10] updated 


\section{References}

[1] J. Lefrancois, Rapporteur talk "Precision tests of the Standard Model" EPS-HEP Conference, Marseille

[2] R. Abela et al., Phys.Lett. B105 (1981) 263

[3] T. Yamazaki et al., Neutrino 84, eds. E.K. Kleinknecht and E.A. Paschos, World Scientific, Singapore (1984) 183

[4] BEBC Collab., A. M. Cooper-Sarkar et al., Phys.Lett. B160 (1985) 207

[5] CHARM Collab., J. Dorenbosch et al., Phys.Lett. B166 (1986) 473

[6] M.E. Duffy et al., Phys.Rev.D38 (1988) 2032

[7] L3 Collab., O. Adriani et al., Phys.Lett. B295 (1992) 371

[8] CCFR-Collab., S.R. Mishra et al., Phys.Rev.Lett.59 (1987) 1397

[9] CHARM II Collab., D. Geiregat et al., Nucl.Instr. and Meth. A325 (1993) 92

[10] CHARM Collab., K. De Winter et al., Nucl.Instr. and Meth. A278 (1989) 670

[11] M. Gronau, C.N. Leung, J.L. Rosner, Phys.Rev.D29 (1984) 2539, Diploma thesis S. Petrak, FU-Berlin, Sept. 1994. 